\title{
Human Intervention and Interface Design in Automation Systems
}

\author{
P. Ponsa, R. Vilanova, B. Amante
}

\author{
Pere Ponsa (corresponding author) \\ Automatic Control Department \\ Technical University of Catalonia \\ EPSEVG, Av. Víctor Balaguer, \\ 08800 Vilanova i la Geltrú, Barcelona, Spain \\ E-mail: pedro.ponsa@upc.edu
}

\section{Ramón Vilanova}

Department de Telecomunicació i Enginyeria de Sistemes

Universitat Autònoma de Barcelona

08193, Bellaterra, Spain

E-mail: ramon.vilanova@uab.cat

\author{
Beatriz Amante \\ Project Department \\ Technical University of Catalonia \\ ETSEIAT Edifici TR5, C. Colom 11, \\ 08222, Barcelona, Spain \\ E-mail: beatriz.amante@upc.edu
}

\begin{abstract}
Human-Machine-Interfaces are with no doubt one of the constitutive parts of an automation system. However, it is not till recently that they have received appropriate attention. It is because of a major concern about aspects related to maintenance, safety, achieve operator awareness, etc has been gained. Even there are in the market software solutions that allow for the design of efficient and complex interaction systems, it is not widespread the use of a rational design of the overall interface system, especially for large scale systems where the monitoring and supervision systems may include hundreds of interfacing screens. It is on this respect hat this communication provides an example of such development also by showing how to include the automation level operational modes into the interfacing system. Another important aspect is how the human operator can enter the control loop in different ways, and such interaction needs to be considered as an integral part of the automation procedure as well as the communication of the automation device.In this paper the application of design and operational modes guidelines in automation are presented inside an educational flexible manufacturing system case study.
\end{abstract}

Keywords: human-automation interaction, process control, display design.

\section{Introduction}

Automation can refer to open-loop operation on the environment or closed-loop control. And the human intervention adopts diverse possibilities: human in the loop (human intervention), human out of the loop (controller intervention) and human on the loop (supervisory control mode over the controlled process). The basic automation replaces the human manual control by an automatic controller; however in highly automated systems is necessary human beings for supervision, adjustment, maintenance, expansion and improvement. Automation increases complexity. It is difficult to maintain operational skills in a automated environment with the 
presence of an abnormal situation when the operator is required to intervene. The complexity of industrial human process supervision makes it necessary to supplement the Human-Computer Interaction approach with a cross-disciplinary cooperation in order to integrate knowledge and methods from other fields, especially Automation and Artificial Intelligence [1].

Our view is that complete control systems engineering must encompass all these approaches. This increasing complexity of production systems has also translated to the automation level. The need to face for larger amounts of information and the capability to interact with other subsystems of the production chain requires of the application of appropriate modelling methodologies. On that respect it is worth to mention that, on the academic side, the authors have developed different tools to tackle such problems.

What is proposed in this paper, and presented by its application to a laboratory scale Flexible Manufacturing System (FMS), is a complete integrated approach for the design of the HMI (Human Machine Interface) system. The development follows a top-down approach where the different screens that constitute the overall system are conceived and particular methods are used to ensure, within each one of the designed interfaces, appropriate ergonomic usage. One of the most interesting points is the introduction, at the automation level, of considerations related to the different start and stop modes of the process units. The inclusion of such considerations at HMI level will help in achieving a more solid and helpful interaction system on its relation with the safety and maintenance operations.

The structure of the paper is as follows. The second section is a brief introduction about the computer architecture, the plant layout in the flexible manufacturing system and brief comments about human-systems interaction. In the next section, the translation from the physical layout to the global design of the HMI is proposed: the human can use the HMI inside a control room or near the machine/process. Section four shows explain the relationship between automatic control and human intervention in manual mode. The paper ends by drawing some concluding remarks.

\section{Human role in the Flexible manufacturing system}

The application of the proposed integrated approach for HMI design is peformed within a concrete educational frame whose base focuses on two subjects from the Automatics and Electronics Engineering degree program: Modelling and Simulating Systems and Integrated Production Systems, at the Technical University of Catalonia UPC, Spain, and Automatic Control and Industrial Informatics at the "Universitat Autňnoma de Barcelona" UAB, Spain. The subjects from the technical engineering program make a special point on technical topics of programmable logic controllers (PLC) and industrial handler robots. In the remaining subjects of the engineering program, Petri Net modelling, simulation by means of the ARENAŠ discrete-even simulation software [2], physical distribution of flexible manufacturing systems and production system modelling and simulation are the main topics. Here, we focus on training the students with system behaviour analysis and simulation software so that they would therefore be able to apply all these techniques in their professional life. This software allows Flow Shop, Job Shop and Flexible Manufacturing Systems simulation [3]. Practical exercises in laboratory complement the theory and, with this purpose, a laboratory scale FMS has been built up, configured and being operative during the last two years which allows the comparison between simulated models and real plant performance. This paper does not describe more technical details of the role playing 
methodology and the project-based learning approach, readers interested in more detail about these techniques are directed to prior work Ponsa et al. [4].

Another step in a foreseeable future is the integration of supervision tools (supervisory control and data acquisition;SCADA software), management tools (Manufacturing Execution Systems; MES; and Enterprise Resource Planning; ERP) as well as production planning tools (planning policy analysis). In this sense it would also be advisable a new subject on automated production management within the study program which integrates MES and ERP systems at the highest level of the Computer Integrated Manufacturing CIM pyramid.

The Figure 1 shows the physical distribution of stations in the above referred educational FMS, the computers architecture and the human intervention with the FMS system: at the top level (human-computer interaction and human in the supervisory task; in the bottom level (human-machine interaction and human in manual mode task). The FMS is composed of electropneumatic units controlled by PLC's and PC's. The main purpose here consists of emulating current manufacturing systems: object mechanization and supply, transfer, product assembling, quality control, checking and storage; and technologies, such as, pneumatics, robotics, PLC, monitoring and production supervision come together. A total of 5 stations constitute the FMS.

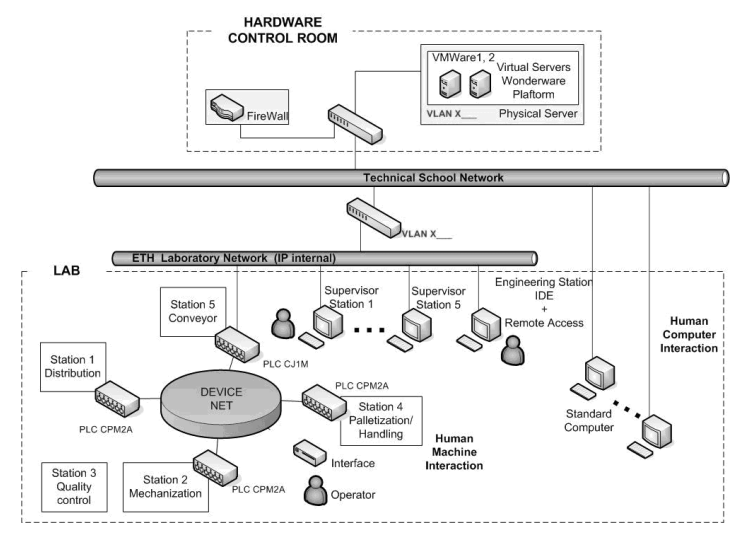

Figure 1: Human intervention in the flexible manufacturing system: high level (supervisory task), low level (machine operation

The cell manufactures two types of products: Product 1 goes across stations 1, 2, 3 and 4 (Palletization); and Product 2 goes from station 4 (Assembling) to 3 and comes back to station 4 (Palletization). The FMS system has product and process flexibility. The Product 1 has 9 different variations (size, color); the Product 2 has 8 different variations (size, color). The FMS can produce 17 different products. From the point of view of process flexibility, the system can produce only the Product 1, only the Product 2 or both; in this last case Station 4 performs two tasks (Assembling and Palletization) but only has a robot so that production planning policy is important in order to prevent an excessive workload in this station.

In contrast with other educational manufacturing systems which use pallets as functional trays for the same purposes, piece transport is carried out directly on an item by item way over the conveyor system from one station to the next one,. At cell control level, we make use of different programmable logic controllers(PLC's) such as the CP2MA, CJ1M models from OMRON; industrial communication protocols (RS-232, RS-422, PC-link, Device Net, Ethernet), and a PLC industrial Network with S7-314 from SIEMENS and communication protocols (RS-485, 
Profibus DP). Regarding programming software which enables local control level and monitoringsupervision linking we are testing products, such as In TouchŠ software and the technological system platform from Wonderware [5]. In a future, the authors want to increase the numbers of the stations and add a storage AS/RS station after the Station 4 [6].

\section{Human machine interface design}

The structure of the HMI application is a distributed interface with six parts (the Main application, and one application for each station) [7]. The Main application has information about the behavior of the flexible manufacturing system: (type of items, Petri Net algorithm, and coordination between stations, maintenance, and safety). The programmable controllers of each station have Device net modules and send this information to the master PLC.

Each station has an autonomous mode of functioning or an integrated mode of functioning. In the first mode, the global design of the HMI is conformed as a set of five autonomous HMI applications. In a near future, one of the problems to solve is the connection between the HMI single applications and works all the FMS in an integrated mode.

The full number of screens of the HMI application for the station1 is 17 screens, while the total number for the global HMI design is of more than 100 screens. Control engineering students have been working in the development of the HMI application for each station. In this sense, expert students (today are working in air traffic control) are the tutors of the novice students. Through the use of project based learning and cooperative learning, the students have made focus groups in order to define a clear and a global structure for all the HMI applications.

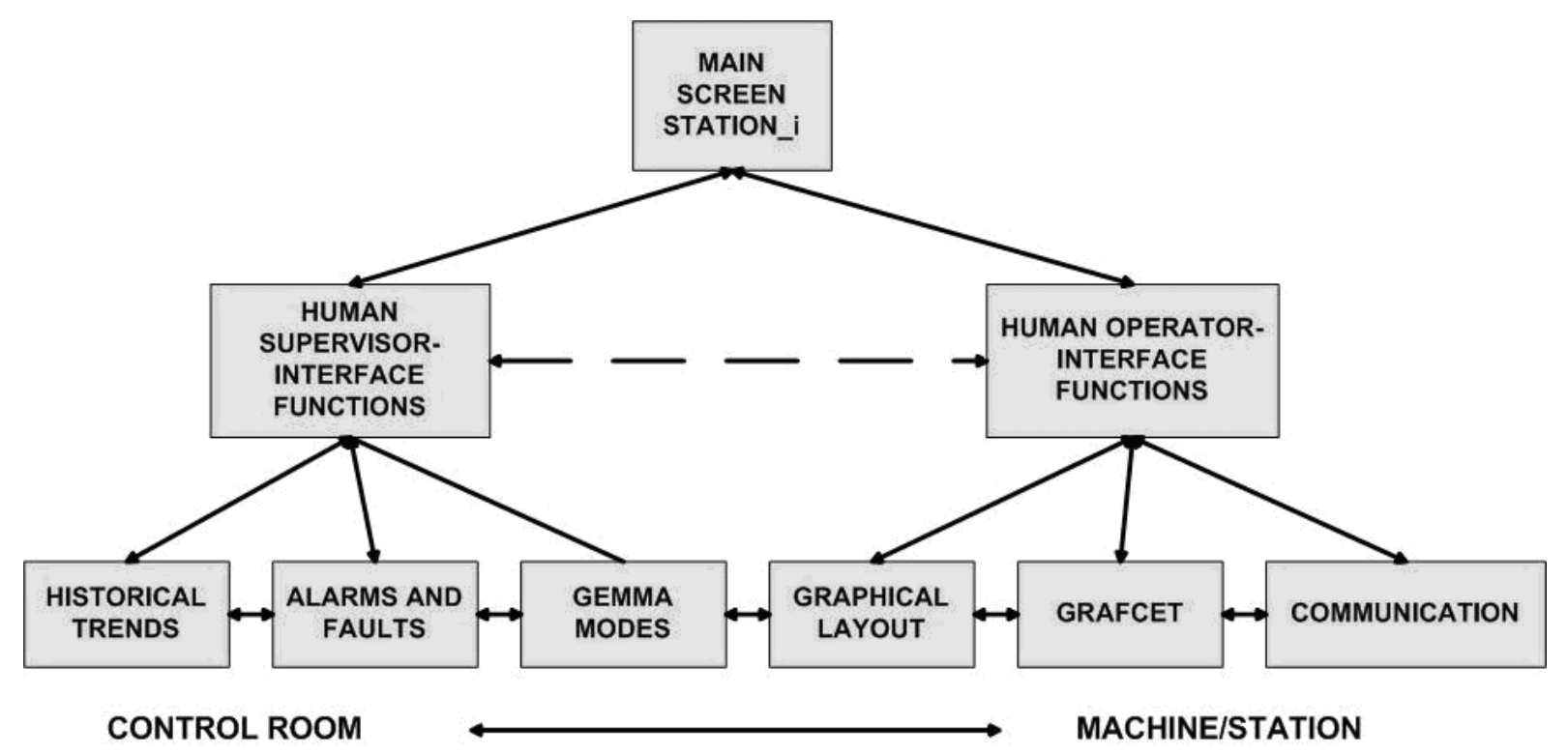

Figure 2: Station_i Display design. Example of a cyclic menu structure. From the main screen to monitoring and supervisory control screens.

In order to achieve an effective HMI application some display design guidelines need to be used. Here the ergonomic display design guideline called GEDIS has been employed [8]. The 
GEDIS guide is a method that seeks to cover all the aspects of the interface design. From the initial point of view of strategies for effective human- computer interaction applied to supervision tasks in an industrial control room [9]. The GEDIS guide offers design recommendations at the time of creating the interface as well as recommendations for improvement of already created interfaces. The GEDIS guide is composed of two parts: description of ten indicators and measure of ten indicators. The indicators have been defined from extracted concepts of other generic human factors guidelines and from aspects of human interface design in human-computer interaction. The method to continue for the use of the GEDIS guide is: analyze the indicator, measure the indicator, obtain the global evaluation index and finally offer recommendations for improvement. For the correct use of the GEDIS guide the collaboration between human factor technicians is necessary since in some cases the expert's opinion is needed to analyze the indicator. This paper does not describe more details of the GEDIS, readers interested in more detail about this guideline are directed to prior work Ponsa and Díaz [10].

For each station we have developed an interface with the same template. The architecture of this interface has two parts: monitoring and supervisory control (see Fig. 2). For monitoring a set of screens (graphical layout of the station, automatic control, communication with the programmable controller PLC) have been defined. The aim of this design is to consider the automatic control loop and the human control. In human supervisory control a set of high level screens (start and stop modes guideline, alarm system, fault detection, diagnostic) has been defined. The aim of this design is to consider the supervisory control loop, a high level over the automatic control loop.

Often, the monitoring interface is associated with the activities of the human operator near the plant, or near the industrial machine (automation level). This task is related to the allocation of physical interface functions and human sensory-motor functions (choose manual or automatic control, activate or deactivate devices, use of the teach pendant of the robot, etc.). With the animating objects properties of the SCADA it is possible to develop a screen with the devices of the station (pneumatic actuators, motors, conveyors and sensors, etc). In this interface it is important the knowledge of the behavior of the devices. In this sense it is important the monitoring interface of station 4. It is a complex interface because it is necessary to develop an animation screen with the activities of the industrial robot (palletization or handling).

Another important screen within the monitoring interface is the panel screen when the human operator can select manual mode or automatic mode. This screen is the one that permits a clear interaction between the human and the automatic controller PLC.

On the other hand the supervisory control interface is associated with the activities of the human operator inside the industrial control room (supervisory control level). The human supervisory task is related to the allocation of interface functions and human cognitive functions (information processing, planning). Respect to the interface this part develops a set of screens: historical trends, alarm screen, fault detection and diagnosis screen. It is necessary to apply a general framework to define which is the correct interface representation of a risky situation. However, each station has specific devices so a specific framework is necessary in order to define a possible anomaly of the station. 


\section{Human intervention in the automation cycle}

Petri Nets (PN) has proved to be a successful approach on a broad range of applications [11], [12], [13]. There is however one point that it is not clear how to deal with by using the PN formalism: the introduction of the human operator. Effectively, in every automation problem the fully automated part is just one part of the solution. It is customary that the operator can enter the loop in different ways, for example by tuning a PID controller [14] and such interaction needs to be considered as an integral part of the automation procedure as well as the communication of the automation device - usually a (PLC) - with the operator. A Human Machine Interface (either as a PC display, Industrial Panel, etc) provides the connection between the human operator action and the input to the control algorithm inside the controller device (control based PC, control based PLC).

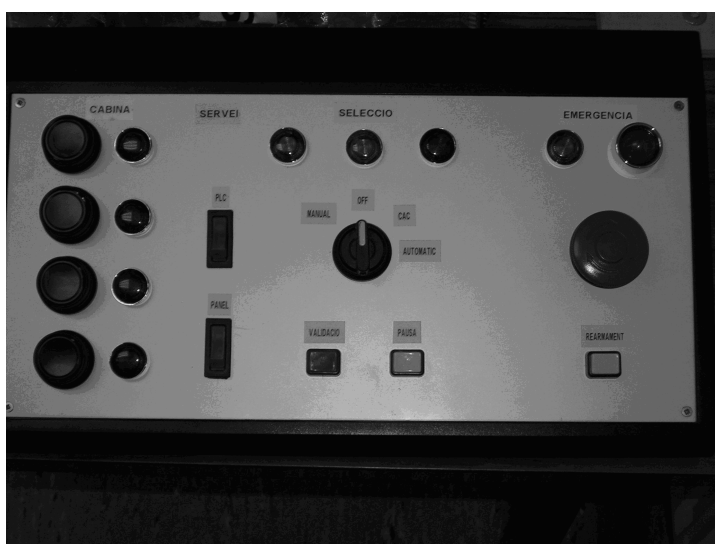

Figure 3: The human operator can intervene inside the automation cycle (GRAFCET transition) with the use of an industrial panel. In this panel the human operator follow the functional operational modes of the GEMMA guide.

On the other side, the industrial counterpart said that other approaches than PN are currently in use. Effectively, even PN allow tackling really large and complex problems, other approaches like Sequential Flow Charts; SFC; or State-Transition Graph; GRAFCET; can be considered the primary automation design tool found on the industry. In fact, some of the PLCs allow direct programming by using GRAFCET [15]. Therefore it seems there is a gap between both of these approaches. What we would like to point out here is that both disciplines should be combined and used. The important point with GRAFCET; a simpler approach if compared with $\mathrm{PN}$; is that it has a close and clear connection with the design guide for start and stop modes called GEMMA [16]. Even the GEMMA guide was started to be used twenty-eight years ago and it was introduced to the engineering students in several places, in our opinion we don't paid sufficiently attention to the GEMMA guide. This is the reason this section would like to focus on the advantages of using the GEMMA guide and using it inside the HMI application explained in the previous section. Although GRAFCET is very useful in describing the detailed operation of a sequential control performed by a PLC it does not provide a general approach for the operation of an automated machine. It is still necessary to define general operational modes and conditions. This is usually done at the specifications definition stage. In this sense, GEMMA is a recommended tool for this task [17].

On the other hand, it has always been difficult in terms of vocabulary to clearly, and precisely, 
explain how to start with manual mode or a semi-automatic mode or an automatic mode. Also what are the consequences of an emergency stop, a safety stop or a fast stop for a production machine? Usually, these questions are answered by each designer in relation to his own experience and knowledge but can seldom be related to a systematical analysis, except in large companies which have been able to define design guide and standards for control and instrumentation.

From the previous observations, the GEMMA guide is a valuable tool to introduce automation in a more general setting than only for the automation of the process production cycle. It is really important to understand the role played by the operator and how he interacts with the automated system [18]. The different operation modes need to be established and be interrelated in a clear and well defined way.

For example in normal conditions the automation cycle is represented by three GEMMA modes: from A1 Initial State Stop, then F1 Normal Production, and A2 Requested Stop at the End of the Cycle and finally comeback to A1. In abnormal situation the automation cycle include the management of an emergency situation: from A1 Initial State Stop, then F1 Normal Production, D1 Emergency Stop, A5 Preparation to Restart after a Failure, A6 Production Reset to the Initital State and finally A1 again.

The main contribution of this section is to advance one step into an integral conception of the automation process: the design of the automation system has to include considerations on operational modes and these have to be reflected in the human interface. With this aim, Fig. 4 shows the GEMMA graphical representation inside the HMI application. When the system is in normal state or in anomaly, the human operator can see an active mode on screen, this facilities the situation awareness.

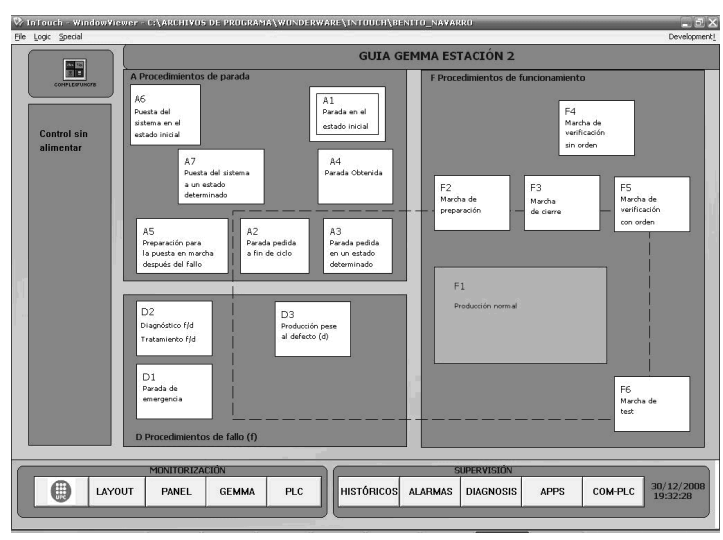

Figure 4: GEMMA graphical representation inside the HMI application. The human operator can observe the changes from Production, Stop or Failure procedures.

When the system is in normal state or in anomaly, the human operator can see an active mode on screen, this facilities the situation awareness.

In the HMI application there is a clear relationship between maintenance and safety services of the system. Inside the PLC an emergency GRAFCET has been designed with instructions in case of emergency. The maintenance recommendations are used in order to reduce the stop of the station, and the stop of the production. The translation of the GEMMA guide inside the PLC needs the use of the GRAFCET representation. When a problem appears in station3, for example, the human operator can activate the emergency stop: in this moment stations 1, 2 and 

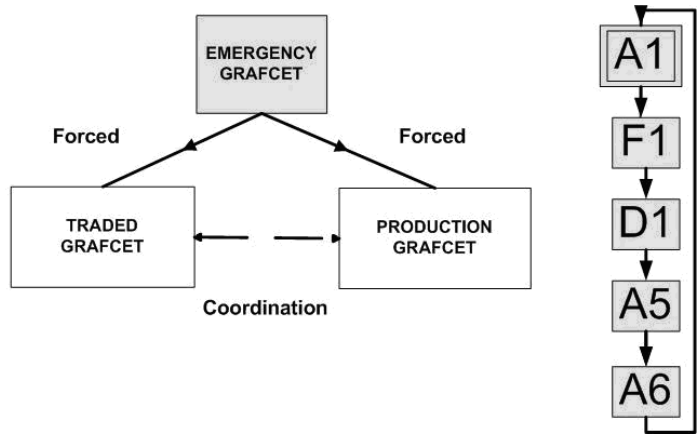

Figure 5: When the emergency stop is activated the Emergency GRAFCET forced the stop of the other GRAFCET. In the Traded GRAFCET we have automatic control or human manual control. The Production GRAFCET is the set of machine operations.

3 stop their production while station 4 and transport unit will still be functioning. When the human operator solves the problem, can procede to activate the reset of the system, drive the system to a safety state and begin again the production in stations 1, 2 and 3 .

\section{Conclusions}

In this work we raised the need for a top-down integrated design for Human Automation systems, with special emphasis on the use of appropriate design and operational guidelines (GEDIS, GEMMA). Different actuation levels are first identified going from the Flexible Manufacturing System level to the automation level at each station. While the interfacing system at the station or global level has to cover, mostly, high level monitoring actions, it is at the automation level where more complex situations may arise. At this point the authors propose to base the use of the start and stop modes guide, GEMMA guide, to build up a convenient representation of the operational stages of the production machines. By designing the automation level HMI along these lines its integration with the automation control system becomes almost natural. The GEMMA guide approach is therefore a recommended taxonomy approach for the introduction of the human operator into the automation cycle in complex academic/industrial domains. It provides a natural relationship between the design of the automation system and the operational modes that are to be considered from the industrial panel point of view.

\section{Acknowledgment}

This work has received financial support from an internal grant of the Technical University of Catalonia (Project: Human centred design in human supervisory control, 2008) and from the Spanish CICYT program under grant DPI2007.63356.

\section{Bibliography}

[1] T. Sheridan and P. Parasuraman, "Human automation interaction," Reviews of Human Factors and Ergonomics, vol. 1, pp. 89-129, 2006.

[2] Rockwell, "Arena simulation software," In URL: http://www.arenasimulation.com, 2009. 
[3] H. Boukef, M. Benrejeb, and P. Borne, "A proposed genetic algorithm coding for flow-shop scheduling problems," International Journal of Computers, Communications and Control, no. 3, pp. 229-240, 2007.

[4] P. Ponsa, B. Amante, J. Roman, S. Oliver, M.Díaz, and J. Vives, "Higher education challenges: introduction of active methodologies in engineering curricula," International Journal of Engineering Education, vol. 25, no. 4, pp. 799-813, 2009.

[5] Wonderware, "Wonderware intouch hmi," In URL: http://global.wonderware.com, 2009.

[6] F. Véliz and G. Lefranc, "Improvement and extension of virtual reality for flexible systems of manufacture," International Journal of Computers, Communications and Control, no. 2, pp. 95-101, 2006.

[7] P. Ponsa, R. Vilanova, and B. Amante, "Towards integral human-machine system conception: from automation design to usability concerns," HSI 2009 - Human System Interaction 2nd International Conference, Catania, Italy, 2009.

[8] P. Ponsa, R. Vilanova, M. Díaz, and A. Gomŕ, "Application of a guideline for design improvement in supervisory control," International Journal on Human.Computer Interaction, vol. 1, pp. 21-36, 2007.

[9] B. Schneiderman, Designing the user interface. Strategies for effective human.computer interaction. Addison-Wesley, third edition, 1997.

[10] P. Ponsa and M. Díaz, "Application of an ergonomic guideline for sugar mill control room interface design," FAIM , 17th International Conference on Factory Automation and Intelligent Manufacturing, Philadelphia, USA, vol. 1, pp. 89-129, 2007.

[11] K. Kurihara, S. Takigawa, N. Nishiuchi, and M. Kitaoka, "Factory automation control software designing method based on petri nets," International Journal of Production Research, vol. 40, no. 15, pp. 3605-3625, 2002.

[12] E. Gutuleac, "Descriptive time membrane petri nets for modeling of parallel computing," International Journal of Computers, Communications and Control, no. 3, pp. 33-39, 2006.

[13] F. Córdova, L. Canete, and L. Q. F. Yanine, "An intelligent supervising system for the operation of an underground mine," International Journal of Computers, Communications and Control, no. 4, pp. 259-269, 2008.

[14] R. Vilanova and O. Arrieta, "Balanced pid tuning application to series cascade control systems," International Journal of Computers, Communications and Control, no. 4, pp. 521$525,2008$.

[15] AFCET-ADEPA, "Le grafcet," Second Edition, Toulouse, Opadus, 1995.

[16] ADEPA, "Le gemma. guide d'etude des modes de marches et d'arrets," Montrouge, ADEPA, 1981.

[17] P. Ponsa and R. Vilanova, Automatización de procesos mediante la guía GEMMA. Edicions UPC, no. 105, 2005.

[18] K. Li, S. Thompson, P. Wieringa, and J. Xeng, "A study on the role on human operators in supervisory automation system and its implications," Proceedings of the 4 th World Intelligent Control and Automation, pp. 3288-3293, 2002. 